\title{
Probabilistic Landslide risk assessment - Application to Eastern Rif (Morocco)
}

\section{Evaluation probabiliste du risque glissement de terrain - Application au Rif oriental du Maroc}

\author{
RFIFI Mohamed ${ }^{1, \mathrm{a}}$, AIT BRAHIM Lahsen ${ }^{1}$ \\ ${ }^{1}$ Université Mohammed V, Faculté des sciences, Unité de recherche GEORISK, LGRN, 4 Avenue Ibn Battouta -Agdal, BP \\ 1014, Rabat, Maroc.
}

\begin{abstract}
The present study is consecrated to the probabilistic mapping of the landslide risk at the local scale of an area that belongs to Al Hoceima city in the western Rif of Morocco. The study focuses mainly on the spatial analysis of multi sources data by using an environment GIS (Geographic Information Systems), and the application of the bivariate probabilistic model to qualify the risk susceptibility. The employed methodology is based on three stages. First, the evaluation of landslide susceptibility (S) by the analysis model cited before. Second, the identification and the estimation of the potential consequences (C) for the existing issues. Finally, the landslide risk $(\mathrm{R})$ is evaluated by combining the susceptibility and the potential consequences map.

This study requires the use of spatial analysis techniques. It also refers to the risk maps scale, generally reduced and being inappropriate at the urban project area. The obtained risk map defines four risk intensities with a spatial resolution of two meters.
\end{abstract}

Keywords: Landslide - Probabilistic mapping - Susceptibility - Consequences - Risk - GIS.

\section{Introduction}

Les risques naturels constituent l'un des principaux enjeux d'aménagement, en particulier dans la région du Rif oriental marocain, zone caractérisée par des paramètres de prédisposition au risque. Cette étude a été initiée à partir d'un constat terrain et un intérêt scientifique initié par des travaux de recherches antérieurs.

Les mouvements de terrain dans le Rif ont fait l'objet de plusieurs travaux, dont ceux de [1] qui demeure l'un des pionniers dans l'étude de ces mouvements. Dans d'autres travaux [2,3] consacrés à des régions précises du Rif, ont invoqué la combinaison de plusieurs facteurs provoquant cet instabilité.

L'objectif principal du présent travail est de mettre en évidence les relations entre des paramètres de susceptibilité (conditions géologiques, topographiques locales...) et les enjeux existants à l'échelle d'une zone urbanisée du Rif menacées par le risque de glissement de terrain.

\section{Site d'étude}

La zone d'étude est située à l'ouest de la ville d'Al Hoceima (figure 1), elle s'étend sur une superficie approximative de $1800000 \mathrm{~m}^{2}$ et elle appartient au Rif oriental, une chaine montagneuse dont la structure est chevauchante avec une superposition de nappes (divisées en plusieurs domaines : les Ghomarides, les Sebtides et la dorsale calcaire).

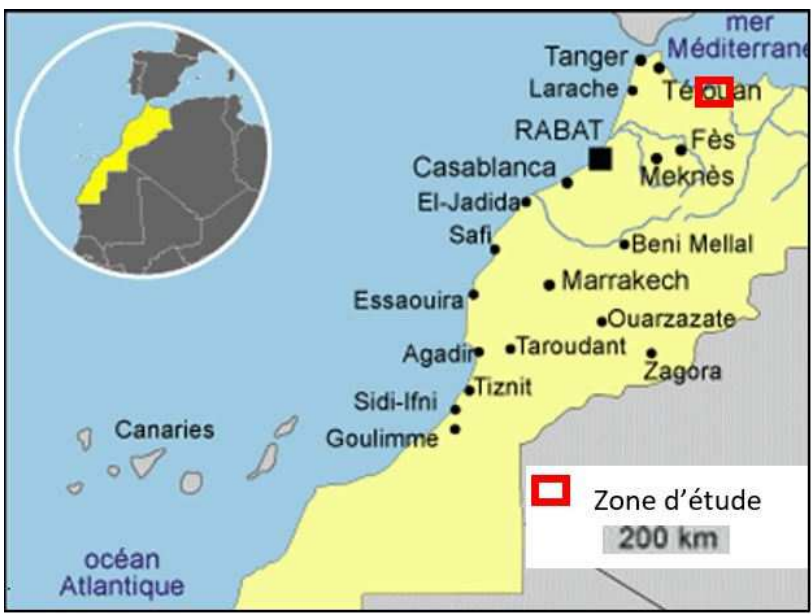

Fig. 1. Situation de la zone d'étude 
Le secteur est marqué par l'affleurement des calcaires et des marnes avec un relief accidenté allant jusqu'à $70^{\circ}$ de pente.

La zone est caractérisée également par une activité sismique remarquable. Plusieurs séismes ont secoués la région, notamment celui de 1994, 2004 et les derniers de 2016.

Le climat dominant la région est de nature semi-aride avec une moyenne de précipitations de $300 \mathrm{~mm} / \mathrm{an}$ et une température moyenne annuelle égale à $18^{\circ} \mathrm{C}$. Plusieurs facteurs ont favorisé le développement du réseau hydrographique dans la zone (formations géologiques, pente du terrain et surface de drainage).

\section{Approche d'analyse du risque employée}

\subsection{Concepts de l'approche}

- L'évaluation de l'ampleur du risque glissement de terrain consiste à exprimer le lien entre la susceptibilité et les conséquences du risque sur les enjeux exposés ;

- La susceptibilité est la probabilité spatiale qu'un glissement de terrain se produit sur un territoire pour différentes conditions environnementales locales. Les techniques d'analyse spatiale [4,5] permettent d'identifier des zones susceptibles selon deux approches: directe (qualitative) et indirecte (probabiliste). L'approche indirecte est la plus objective et permet d'obtenir des cartes de susceptibilité plus fiables et réalistes. Elle est retenue dans le cadre de ce travail.

- Le principe de l'approche indirecte «bi-variée» consiste à définir les relations mathématiques entre les facteurs de prédisposition (Variables indépendantes) et l'occurrence spatiale du glissement (variable dépendante).

- La définition des conséquences du risque consiste à évaluer une valeur d'endommagement pour chaque élément exposé.

- Le calcul du risque est effectué par croisant, à l'aide d'un tableau à double entrées [6], des classes de susceptibilité et les classes de conséquences potentielles simulées.

\subsection{Données de l'étude}

Les données pour l'analyse de la susceptibilité peuvent être regroupées en cinq groupes de variables (table 1) : inventaire géomorphologique des glissements de terrain observés, pente topographiques, géologie, formations superficielles, hydrographique et occupation du sol.

Table 1. Données analysées pour l'évaluation de la susceptibilité

\begin{tabular}{|c|l|c|}
\hline \multicolumn{2}{|c|}{ Variable dépendante/explicative } & Source \\
\hline Vd & Glissement de terrain (GT) & Observations, cartes \\
\hline & Pente (PE) & Modèle numérique de \\
\hline
\end{tabular}

\begin{tabular}{|l|l|l|}
\hline \multirow{4}{*}{ Ve } & & terrain \\
\cline { 2 - 3 } & Lithologie (LI) & $\begin{array}{l}\text { Carte géologique et } \\
\text { rapports }\end{array}$ \\
\cline { 2 - 3 } & Formations superficielles (FS) & Réseau hydrographique (HY) \\
& $\begin{array}{l}\text { Cartes et modèle } \\
\text { numérique de terrain }\end{array}$ \\
\cline { 2 - 3 } & Occupation du sol (OS) & Cartes \\
\hline
\end{tabular}

L'inventaire géomorphologique des glissements de terrain a été réalisé à partir de l'observation terrain et les rapports techniques archivés (figure 2).

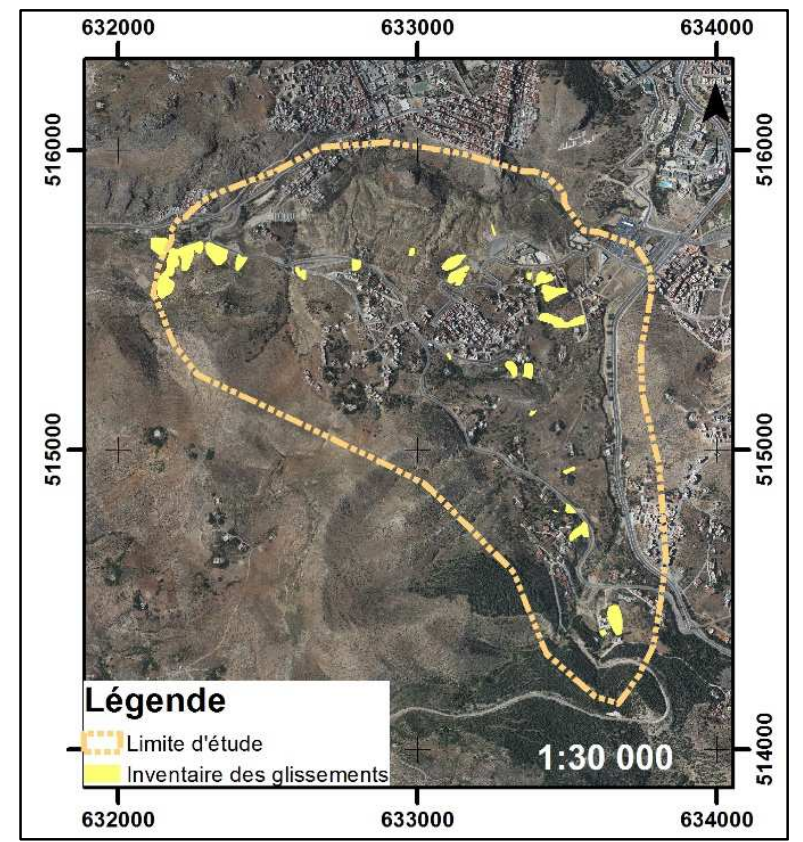

Fig. 2. Carte de l'inventaire géomorphologique des glissements de terrain actifs

Les valeurs attribuées pour qualifier le degré de l'endommagement (CEP) en cas de glissement reflète le cout des dommages monétaires et/ou humains engendrés par le phénomène. Les valeurs ont été quantifiées par retour d'expérience dans des travaux antérieurs [7].

\subsection{Cartographie de la susceptibilité}

La cartographie de la susceptibilité consiste à :

- Etablir un inventaire cartographique des zones d'occurrence des glissements ;

- Tester l'indépendance conditionnelle (test $\chi 2$ ) entre les cinq variables indépendantes pour in seuil de signification $\alpha=0.05$ [8] ;

- Définir les combinaisons entre les variables indépendantes (au sens statistique). Suivant le protocole défini par [9] ;

- Valider statistiquement la combinaison employée en introduisant les $2 / 3$ de la population totale des glissements de terrain, à partir d'un tirage aléatoire ;

- Caler le modèle obtenu en utilisant le $1 / 3$ de la population des glissements restante ;

- Interpréter les résultats du modèle sous forme de classes de susceptibilité (S). Le choix des classes est établi par discrétisation manuelle, basée sur l'existence de seuils naturels visibles sur la courbe cumulative des valeurs de susceptibilité. 


\subsection{Cartographie de conséquences}

L'évaluation est faite par identification des catégories des enjeux existants (structurels, corporels et fonctionnels) et par attribution des coefficients d'endommagement (CEP) à chaque détail exposé à l'enjeu. La valeur du coefficient est comprise entre 0 et 1 (table 2).

Table 2. Enjeux identifiés au niveau de la zone et valeurs du coefficient d'endommagement (CEP) attribuées

\begin{tabular}{|l|l|}
\hline \multicolumn{2}{|c|}{ ENJEU CORPOREL ET STRUCTUREL (E1) } \\
\hline Type de zone & CEP \\
\hline Urbaine & 1,0 \\
\hline Naturelle & 0,3 \\
\hline Affleurement rocheux et sol nu & 0,1 \\
\hline \multicolumn{2}{|c|}{ ENJEU FONCTIONNEL (E2) } \\
\hline Type de l'infrastructure & CEP \\
\hline Route principale & 1,0 \\
\hline Route secondaire & 0,8 \\
\hline Piste & 0,6 \\
\hline Aménagement hydraulique & 1,0 \\
\hline Confortement & 1,0 \\
\hline Murs de soutènement & 1,0 \\
\hline
\end{tabular}

La combinaison des valeurs du CEP pour les enjeux étudiés (E1 et E2) permet de déduire 5 classes de dommages.

\subsection{Cartographie du risque total}

Cette cartographie est obtenue par le croisement des classes de la carte de susceptibilité et de conséquences potentielles suivant une matrice à double entrée (table 3).

Table 3. Croisement de la carte de susceptibilité et la carte de dommages

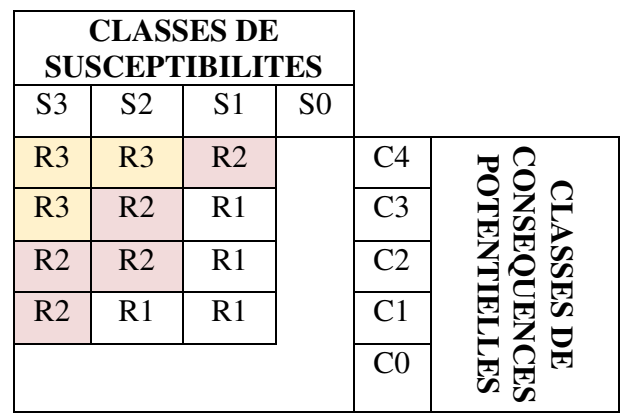

\section{Résultats et interprétation}

\subsection{Carte de susceptibilité finale modélisée}

Les tests effectués pour le calage des variables indépendantes indiquent que la meilleure combinaison est celle qui regroupe les cinq variables, par ordre hiérarchique, la pente, la lithologie, l'occupation du sol, la géologie et la distance aux cours d'eau (FS + LI + OS $+\mathrm{PE}+\mathrm{HY})$.
Une combinaison de trois variables seulement (FS, LI et OC) produit une erreur relative sur les surfaces modélisées de $40 \%$ (figure 3). L'ajout des variables PE et HY permet une réduction de 29\% d'erreur.

La cartographie de la susceptibilité finale en 4 classes a finalement été retenue. Elle identifie respectivement des aires de susceptibilité de $96000 \mathrm{~m}^{2}, 664000 \mathrm{~m}^{2}, 983000$ $\mathrm{m}^{2}$ et $57000 \mathrm{~m}^{2}$ pour $\mathrm{S} 0, \mathrm{~S} 1, \mathrm{~S} 2$ et $\mathrm{S} 3$ (figure 4) :

- Susceptibilité forte (S3) : coïncide avec des terrains marneux de pentes moyennes et fortes exposés à l'action des eaux de surface (3\% de l'aire d'étude) ;

- Susceptibilité modérée (S2): corresponde généralement à des formations marneuses et des encroutements calcaire ayant des pentes moyennes (55\% du secteur) ;

- Susceptibilité faible (S1) et nulle (S0) coïncide avec des terrains de faibles pentes occupant $42 \%$.

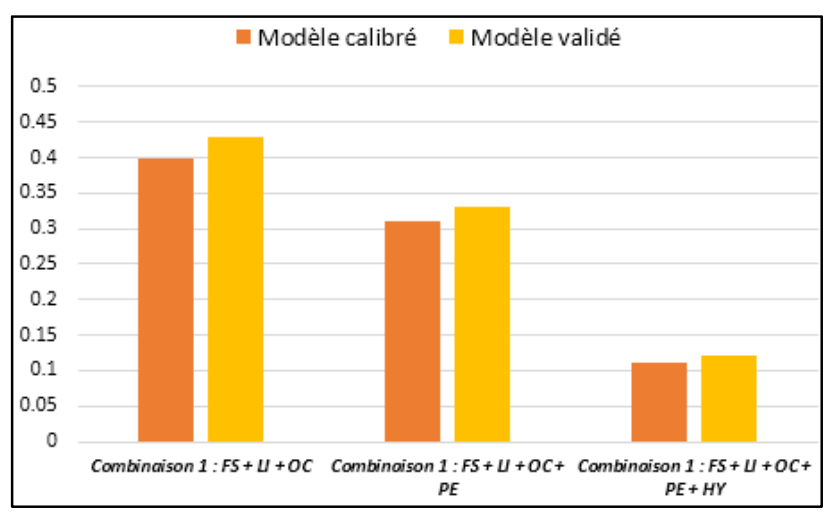

Fig. 3. Combinaison des paramètres de prédispositions au risque et résultats du modèle retrouvés

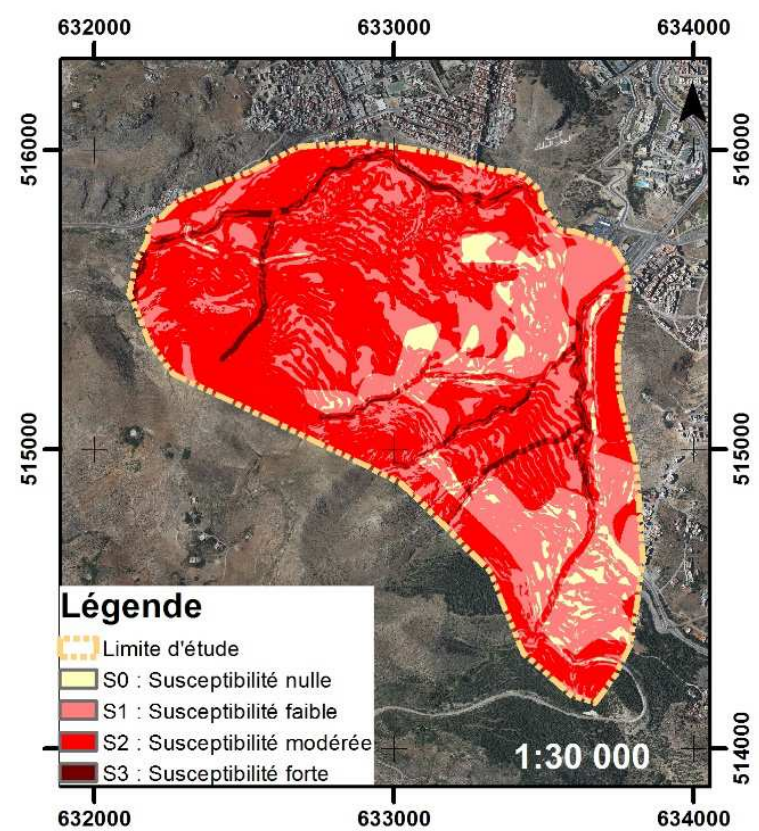

Fig. 4. Carte de susceptibilité aux glissements de terrain

\subsection{Carte des conséquences potentielles}

La carte de dommage met en évidence des enjeux liés aux infrastructures et réseau du transport existant, les zones urbanisées et en urbanisation progressive. La carte 
identifie respectivement des aires de conséquences potentielles de $927000 \mathrm{~m}^{2}, 4000 \mathrm{~m}^{2}, 733000 \mathrm{~m}^{2}, 1000$ $\mathrm{m}^{2}$ et $135000 \mathrm{~m}^{2}$ pour les classes $\mathrm{C} 0, \mathrm{C} 1, \mathrm{C} 2, \mathrm{C} 3$ et $\mathrm{C} 4$ (figure 5).

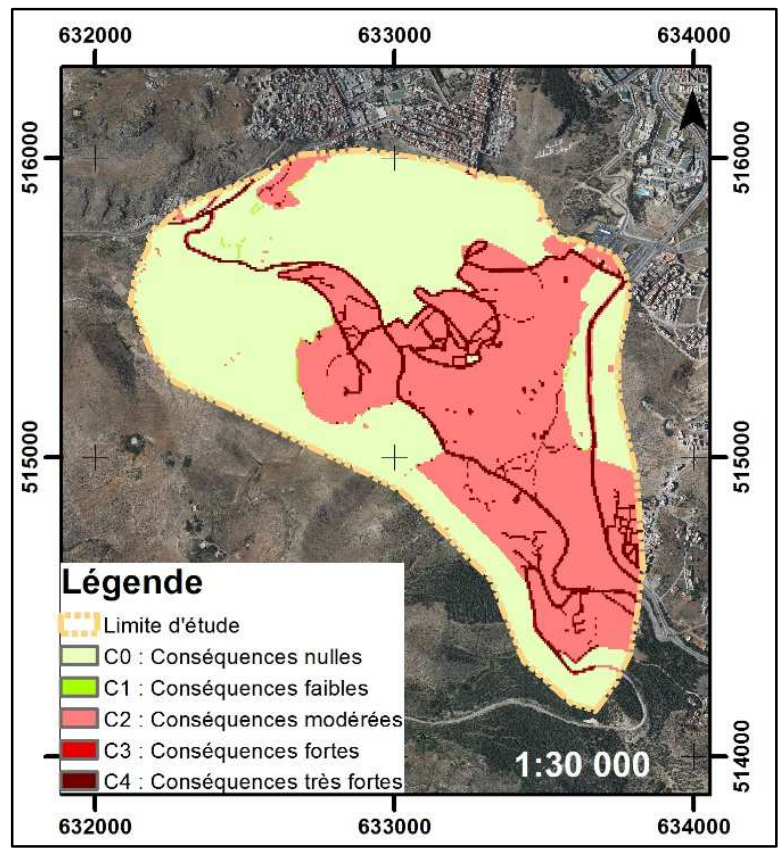

Fig. 5. Carte de conséquences des glissements de terrain

\subsection{Carte du risque total}

Le croisement des cartes de susceptibilité et de conséquences potentielles indique que les zones de risque fort (R3) sont très peu développées sur le territoire étudié (figure 6). Les zones R3 correspondent à des secteurs en urbanisation ayant une pente forte combinée à un écoulement de l'eau de ruissèlement. Le niveau de risque négligeable représente $60 \%$ de la zone $\left(1081000 \mathrm{~m}^{2}\right)$ et correspond aux zones non engagées dans des activités d'aménagement. Cependant, le risque faible et modéré se concentre à l'intérieur de la zone d'étude et ils représentent respectivement $406000 \mathrm{~m}^{2}$ et $294000 \mathrm{~m}^{2}$.

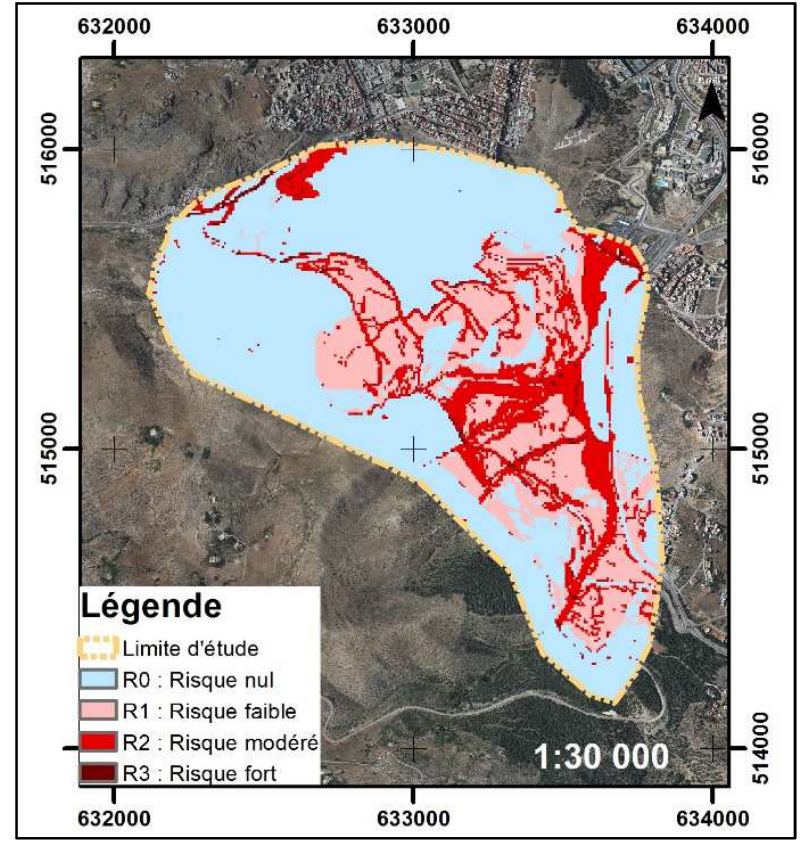

Fig. 6. Carte du risque global des glissements de terrain

\section{Conclusion}

Cette étude s'inscrit dans le cadre de l'analyse du risque «glissement de terrain» et l'évaluation probabiliste de son intensité à l'échelle d'une zone située à l'ouest de la ville d'Al Hoceima. L'étude démontre des niveaux de risques variables déterminés par les techniques d'analyse spatiale. A partir d'une démarche en 3 étapes, le risque est identifié et cartographié en associant plusieurs modèles d'analyse: (1) la susceptibilité de la zone au glissement de terrain est analysée par un modèle probabiliste bi-varié de la théorie d'évidence ; (2) les conséquences potentielles sont estimées par approche semi-empirique ; (3) le risque est évalué en combinant la carte de susceptibilité et celle des conséquences potentielles.

La cartographie du risque de glissement nécessite un inventaire géomorphologique des glissements au niveau de la zone. C'est un travail qui nécessite une bonne connaissance du terrain, une exploitation des rapports techniques disponibles et la mise en place d'une base de données utiles. Le bon modèle retrouvé par analyse probabiliste a démontré des résultats satisfaisants et une bonne adéquation aux particularités du secteur d'étude.

\section{Références}

1. Millies-Lacroix, Les glissements de terrain, présentation d'une carte prévisionnelle des mouvements de terrain dans le Rif. Mine et Géologie, 27, pp. 45-54 (1968)

2. K. Margua, Essai de cartographie des risques naturels : Application à l'aménagement de la région d'Al Hoceima. Thèse universitaire, Univ. FrancheComté, 196 p (1994)

3. A. Talhaoui, M. Aberkan, A. Iben Brahim, A. El Mouraouah, N. Jabour, Contribution à l'étude des risques naturels. Cas de la région d'Al Hoceima. 
Oujda, Colloque des Bassins Sédimentaires Marocains (1999)

4. F. Guzzetti, A. Carrara, M. Cardinali, P. Reichenbach, Landslide hazard evaluation: a review of current techniques and their application in a multi-scale study, Central Italy. Geomorphology, 31, pp. 181-216 (1999)

5. C.J. Van Westen, The modelling of landslide hazard using GIS. Surveys in Geophysics, 21, pp. 241-255 (2000)

6. C. Ko Ko, P. Flentje, R. Chowdhury. Landslides qualitative hazard and risk assessment method and its reliability. Bulletin of Engineering Geology and the Environment, 63, $\mathrm{n}^{\circ} .2$, pp. 149-165 (2004)
7. F. Leone, Concept de vulnérabilité appliqué à l'évaluation des risques générés par les phénomènes de mouvements de terrain. Environnement et société. s.l: Université de Grenoble (1996)

8. G.F. Bonham-Carter, Geographic Information System for Geoscientifists: modelling with GIS. Computer methods in the Geosciences. Pergamon Press, 13 (1994)

9. Y. Thiery, S. Sterlacchini, J.P Malet, A. Puissant, A. Remaître, O. Maquaire, Strategy to Reduce Subjectivity in Landslide Susceptibility Zonation by GIS in Complex Mountainous Environments. Heraklion, Greece: 7th AGILE Conference on GIScience

(2004) 